\title{
Dried Plums, Prunes and Bone Health: A Comprehensive Review
}

\author{
Taylor C. Wallace ${ }^{1,2}$ \\ 1 Department of Nutrition and Food Studies, George Mason University, Fairfax, VA 22030, USA; \\ taylor.wallace@me.com; Tel.: +1-270-839-1776 \\ 2 Think Healthy Group, Inc., Washington, DC 20001, USA
}

Received: 15 March 2017; Accepted: 17 April 2017; Published: 19 April 2017

\begin{abstract}
The 2015-2020 Dietary Guidelines for Americans advocate for increasing fruit intake and replacing energy-dense foods with those that are nutrient-dense. Nutrition across the lifespan is pivotal for the healthy development and maintenance of bone. The National Osteoporosis Foundation estimates that over half of Americans age 50+ have either osteoporosis or low bone mass. Dried plums, also commonly referred to as prunes, have a unique nutrient and dietary bioactive profile and are suggested to exert beneficial effects on bone. To further elucidate and summarize the potential mechanisms and effects of dried plums on bone health, a comprehensive review of the scientific literature was conducted. The PubMed database was searched through 24 January 2017 for all cell, animal, population and clinical studies that examined the effects of dried plums and/or extracts of the former on markers of bone health. Twenty-four studies were included in the review and summarized in table form. The beneficial effects of dried plums on bone health may be in part due to the variety of phenolics present in the fruit. Animal and cell studies suggest that dried plums and/or their extracts enhance bone formation and inhibit bone resorption through their actions on cell signaling pathways that influence osteoblast and osteoclast differentiation. These studies are consistent with clinical studies that show that dried plums may exert beneficial effects on bone mineral density (BMD). Long-term prospective cohort studies using fractures and BMD as primary endpoints are needed to confirm the effects of smaller clinical, animal and mechanistic studies. Clinical and prospective cohort studies in men are also needed, since they represent roughly $29 \%$ of fractures, and likewise, diverse race and ethnic groups. No adverse effects were noted among any of the studies included in this comprehensive review. While the data are not completely consistent, this review suggests that postmenopausal women may safely consume dried plums as part of their fruit intake recommendations given their potential to have protective effects on bone loss.
\end{abstract}

Keywords: dried plum; prune; bone

\section{Introduction}

The 2015-2020 Dietary Guidelines for Americans (DGA) advocate for healthy eating patterns that include a variety of fruits. This includes all fresh, frozen, canned and dried fruits and fruit juices [1]. The recommended intake of fruit in the Healthy US-Style Eating Pattern at the 2000-kcal level is two cup-equivalents of fruit per day. Increasing the amount and variety of fruits Americans consume is a strategy that helps individuals meet a wide range of nutrient requirements. However, per the 2015-2020 DGA, average intake of fruit is well below recommendations for almost all age-sex groups, except in children ages 1-8 years [1]. Average intake of fruit is lowest among girls ages 14-18 years and in women age 51+ years [1], two critical time points in bone development and maintenance.

Osteoporosis is a rising public health concern, given the aging population and suboptimal dietary intakes of dairy, fruits, vegetables and whole grains, which provide a variety of essential 
nutrients that influence bone accretion and maintenance across the lifespan. The National Osteoporosis Foundation estimates that $10.3 \%$ of Americans over the age of 50 years have osteoporosis $(t$-score $\leq 2.5)$, and $43.9 \%$ have low bone mass (also commonly referred to as osteopenia; $t$-score $\leq 1.0$ ), a risk factor for osteoporosis [2]. The risk of fractures increases with age among individuals age 50+, and differs by sex, race and ethnicity [2,3]. Although many factors contribute to this debilitating event, the most significant causes are reduction in bone mass, structural deterioration and increased frequency of falls. It 2005, it was estimated that the over two million incident osteoporotic fractures occurring annually in the U.S. had an economic burden of $\$ 16.9$ billion, which is anticipated grow to three million fractures at a cost of $\$ 25.3$ billion by 2025 [4]. Men account for $29 \%$ of these fractures and $25 \%$ of the cost burden [4]. Optimization of lifestyle factors known to influence bone mass and strength is an important strategy aimed at reducing the risk of fractures later in life.

Plums are a type of drupe fruit that belong to the subgenus Prunus (family Rosaceae). They differ from other subgenera of drupe fruits (cherries, peaches, etc.) since the shoots have a terminal bud and unclustered single side buds, flowers combine in groups of one to five on short stems, the fruit has a crease running down one side and a smooth seed. There are over 40 species of plums currently documented, although two species, the European plum (Prunus domestica) and Japanese plum (Prunus salicina and hybrids) are of commercial significance globally [5]. The origin of European plum is thought to have been near the Caspian Sea, while Japanese plums originated in China, but derived their name from the country where they were cultivated. European plums were introduced in the U.S. by pilgrims in the 17th century, while Japanese plums were introduced to the U.S. in the late 19th century. China, Serbia and Romania are the world's leading producers of plums. Worldwide, greater than 11.2 million metric tons of plums were harvested in 2014 per the Food and Agriculture Organization (FAO) of the United Nations [6]. While all prunes originate from fresh plums, not all plum varieties are considered prunes. Commercialized prunes, also commonly known as dried plums, are the dehydrated version of the cultivar Prunus domestica L. cv d'Agen. This specific variety has a naturally-occurring sugar content that enables it to be dried while still containing the pit, without being fermented. The State of California produces $\sim 99 \%$ of the plums in the U.S. and $\sim 40 \%$ of the world's dried plums [7].

Dried plums are widely known for their laxative effect, which is commonly attributed to their dietary fiber content [8], but is also likely influenced by the significant amounts of phenolics (e.g., chlorogenic acid) and sorbitol present in the fruit. Dried plums are not only a source of dietary fiber, but also a good source of potassium and vitamin K (Table 1). One serving or $\sim 4$ dried plums is 92 kilocalories and provides $2.4 \mathrm{~g}$ of dietary fiber, $280 \mathrm{mg}$ of potassium and $22.8 \mu \mathrm{g}$ of vitamin K. Dried plums also contain several dietary bioactives, including phenolic compounds, such as 3-caffeoylquinic acid, 4-caffeoylquinic acid, 5-caffeoylquinic acid, 3-p-coumarolylquinic acid, caffeic acid, p-coumaric acid and quercetin-3-O-rutinoside [9], whose benefits may extend beyond the basic nutrition requirements of humans. There is an emerging body of evidence from laboratory, animal and human studies that suggests that dried plums may exert an effect on bone health. Hooshmand and others found that two servings (100 g) of dried plums per day slowed the rate of bone turnover and helped to improve bone mineral density (BMD) in a clinical study of 160 randomized postmenopausal women (100 completed the study) not receiving hormone replacement therapy [10]. A more recent clinical study by the same group confirmed the bone protective effects in postmenopausal women receiving one serving of dried plums per day [11]. However, a comprehensive review of dried plums and bone health is not currently present in the peer-reviewed scientific literature. 
Table 1. Nutritional profile of dried plums per $100 \mathrm{~g}$.

\begin{tabular}{|c|c|c|c|}
\hline Nutrient & Unit & DV & Plums, Dried (Prunes) (09291) ${ }^{a}$ \\
\hline \multicolumn{4}{|l|}{ Macronutrients } \\
\hline Water & $\mathrm{g}$ & ND & 30.92 \\
\hline Energy & Kcal & 2000 & 240 \\
\hline Protein & $\mathrm{g}$ & 50 & 2.18 \\
\hline Fat & $\mathrm{g}$ & 78 & 0.38 \\
\hline Carbohydrate & $\mathrm{g}$ & 275 & 63.88 \\
\hline Fiber & $\mathrm{g}$ & 28 & 7.1 \\
\hline \multicolumn{4}{|l|}{ Minerals } \\
\hline Calcium & $\mathrm{mg}$ & 1300 & 43 \\
\hline Iron & $\mathrm{mg}$ & 18 & 0.93 \\
\hline Magnesium & $\mathrm{mg}$ & 400 & 41 \\
\hline Phosphorus & $\mathrm{mg}$ & 1000 & 69 \\
\hline Potassium & $\mathrm{mg}$ & 4700 & 732 \\
\hline Sodium & $\mathrm{mg}$ & 2300 & 2.0 \\
\hline Zinc & $\mathrm{mg}$ & 15 & 0.44 \\
\hline Copper & $\mathrm{mg}$ & 2 & 0.281 \\
\hline Manganese & $\mathrm{mg}$ & 2 & 0.299 \\
\hline Selenium & $\mu \mathrm{g}$ & 70 & 0.3 \\
\hline Vitamins & & & \\
\hline Vitamin C & $\mathrm{mg}$ & 60 & 0.6 \\
\hline Thiamin & $\mathrm{mg}$ & 1.5 & 0.51 \\
\hline Riboflavin & $\mathrm{mg}$ & 1.7 & 0.186 \\
\hline Niacin & $\mathrm{mg}$ & 20 & 1.882 \\
\hline Pantothenic acid & $\mathrm{mg}$ & 10 & 0.422 \\
\hline Vitamin B6 & $\mathrm{mg}$ & 2 & 0.205 \\
\hline Folate & $\mu \mathrm{g}$ & 400 & 4.0 \\
\hline Choline & $\mathrm{mg}$ & 550 & 10.1 \\
\hline Vitamin B12 & $\mu \mathrm{g}$ & 6 & 0.0 \\
\hline Vitamin A & IU & 5000 & 781 \\
\hline Vitamin D & $\mu \mathrm{g}$ & 20 & 0.0 \\
\hline Vitamin E & $\mathrm{mg}$ & 30 & 0.43 \\
\hline Vitamin K & $\mu g$ & 80 & 59.5 \\
\hline
\end{tabular}

ND $=$ not defined by FDA.
Nutrient Database Numb

\section{Methods}

\subsection{Literature Search}

A comprehensive literature search was conducted as of 24 January 2017 using the PubMed database. The search methodology is outlined in Table 2. A systematic literature search was not conducted for lack of clinical and observational evidence and since the focus was to evaluate potential mechanisms from various types of data.

Included in the review were cell, animal, population and clinical studies in the English language that assessed the effects of dried plums or extracts of the former on markers of bone health. All articles were screened by title/abstract and, in some cases, full-text. A complete manual search of reference lists of original studies was also conducted. Excluded studies $(n=26)$ were those of any kind that did not assess dried plum (prune) or plum intake on one or more markers or clinical endpoints of bone health. 
Table 2. Search strategy.

\begin{tabular}{cccc}
\hline Search No. & Search & Results & Search Type \\
\hline 1 & bone and bones (MeSH Terms) & 536,127 & Advanced \\
2 & bone AND (fracture* OR density OR resorption OR development) & 340,163 & Advanced \\
3 & osteoporosis & 73,977 & Advanced \\
4 & osteoblasts & 36,771 & Advanced \\
5 & osteoclasts & 19,477 & Advanced \\
6 & \#1 OR \#2 OR \#3 OR \#4 OR \#5 & 762,438 & Advanced \\
7 & Prune(TIAB) OR plum(TIAB) OR dried plum(tiab) & 2460 & Advanced \\
8 & \#6 AND \#7 & 50 & \\
\hline
\end{tabular}

$\mathrm{MeSH}=$ medical subject heading; TIAB = title/abstract.

\subsection{Data Extraction}

Quantitative and qualitative data information from each study, including author and year of study, geographic study location, study design, product information, intervention, population, markers measured, duration and results, were extracted (Tables 3-5). 
Table 3. Clinical trials.

\begin{tabular}{|c|c|c|c|c|c|c|c|c|}
\hline Reference & Location & Design & Plum Product & Intervention & Population & Markers Measured & Duration & Results \\
\hline $\begin{array}{l}\text { Arjmandi et al. } \\
2002 \text { [12] }\end{array}$ & USA & $\mathrm{RCT}$ & $\begin{array}{l}\text { Dried plum } \\
\text { (P. domestica) }\end{array}$ & $\begin{array}{l}100 \mathrm{~g} / \text { day DP or } \\
75 \mathrm{~g} / \text { day dried } \\
\text { apple }\end{array}$ & $\begin{array}{l}\text { Postmenopausal } \\
\text { women }(n=58)\end{array}$ & $\begin{array}{l}\text { IFG-I, IGFBP-3, AP, } \\
\text { BSAP, TRAP, } \\
\text { phosphorus, } \\
\text { magnesium, calcium, } \\
\text { urine-DPD, urine-HP }\end{array}$ & 3 months & $\begin{array}{l}\text { DP led to borderline significant } \\
\text { increases in AP and IGF-1. } \\
\text { Borderline significant increase } \\
\text { in BSAP No significant } \\
\text { differences on other markers } \\
\text { measured. }\end{array}$ \\
\hline $\begin{array}{l}\text { Hooshmand et al. } \\
2011 \text { [10]; } \\
\text { Hooshmand et al. } \\
2014 \text { [13] }\end{array}$ & USA & $\mathrm{RCT}$ & $\begin{array}{l}\text { Dried plum } \\
\text { (P. domestica) }\end{array}$ & $\begin{array}{l}100 \mathrm{~g} / \text { day DP or } \\
75 \mathrm{~g} / \text { day dried } \\
\text { apple }\end{array}$ & $\begin{array}{l}\text { Postmenopausal } \\
\text { women with osteopenia } \\
(n=160 \text { enrolled; } \\
100 \text { completed })\end{array}$ & $\begin{array}{l}\text { BMD (spine, ulna, } \\
\text { total hip and whole } \\
\text { body), RANKL, OPG, } \\
\text { sclerostin, osteocalcin, } \\
\text { TRAP-5b, BALP, DPD, } \\
\text { phosphorus, calcium }\end{array}$ & 1 year & $\begin{array}{l}\text { Significant increase in BMD at } \\
\text { the spine and ulna in both } \\
\text { groups, however increases } \\
\text { were significantly greater in } \\
\text { the DP group compared to } \\
\text { dried apple control. Borderline } \\
\text { significant increase in RANKL } \\
\text { and OPG on DP group. } \\
\text { Significant decrease in } \\
\text { sclerostin, BSAP and TRAP-5b. } \\
\text { No significant differences on } \\
\text { other markers measured. }\end{array}$ \\
\hline $\begin{array}{l}\text { Simonthsnavice } \\
\text { et al. } 2014 \text { [14] }\end{array}$ & USA & Intervention & $\begin{array}{l}\text { Dried plum } \\
\text { (P. domestica) }\end{array}$ & $\begin{array}{l}90 \mathrm{~g} / \text { day DP with } \\
\text { combination } \\
\text { resistance training } \\
\text { vs. resistance } \\
\text { training alone. }\end{array}$ & $\begin{array}{l}\text { Female breast cancer } \\
\text { survivors }(n=23)\end{array}$ & $\begin{array}{l}\text { BMD (lumbar spine, } \\
\text { femur and forearm), } \\
\text { TRAP-5b, BSAP, CRP }\end{array}$ & 6 months & $\begin{array}{l}\text { No significant differences } \\
\text { between groups or any } \\
\text { group-by-time interaction. }\end{array}$ \\
\hline $\begin{array}{l}\text { Hooshmand } \\
\text { et al. } 2016 \text { [11] }\end{array}$ & USA & $\mathrm{RCT}$ & $\begin{array}{l}\text { Dried plum }(P . \\
\text { domestica) }\end{array}$ & $\begin{array}{l}0,50 \mathrm{~g} / \text { day or } \\
100 \mathrm{~g} / \text { day DP. }\end{array}$ & $\begin{array}{l}\text { Postmenopausal } \\
\text { women }(n=48)\end{array}$ & $\begin{array}{l}\text { BMD (total body, total } \\
\text { hip, L1-L4 and ulna), } \\
\text { BAP, TRAP-5b, } \\
\text { BAP/TRAP-5b ratio, } \\
\text { hs-CRP, IGF-1, } \\
\text { sclerostin, RANKL, } \\
\text { OPG, RANKL/OPG } \\
\text { ratio, 25(OH)D, } \\
\text { calcium, phosphorus }\end{array}$ & 6 months & $\begin{array}{l}\text { Compared to controls: Both } \\
\text { doses of DP prevented loss of } \\
\text { total body BMD but not hip, } \\
\text { spine or ulna BMD as } \\
\text { compared to the control group. } \\
\text { TRAP-5b decreased at } 3 \\
\text { months and this was sustained } \\
\text { at } 6 \text { months in both } 50 \text { and } 100 \\
\text { g DP groups. BAP/TRAP-5b } \\
\text { ratio was significantly greater } \\
\text { at } 6 \text { months in both DP groups. } \\
\text { No significant differences on } \\
\text { other markers measured. }\end{array}$ \\
\hline
\end{tabular}

25(OH)D = 25-hydroxyvitamin D; AP = alkaline phosphatase; BAP = bone alkaline phosphatase; $\mathrm{BMD}=$ bone mineral density; $\mathrm{BSAP}=$ bone-specific alkaline phosphatase; $\mathrm{DP}=$ dried plums; DPD = deoxypyridinoline; HS = hydroxylysylpyridinoline; hs-CRP = high sensitivity C-reactive protein; IGF-1 = insulin-like growth factor-1; IGFBP-3 = insulin-like growth factor-binding protein-3; OPG = osteoprotegerin; RANKL = receptor activator of nuclear factor kappa-B ligand; TRAP = tartrate-resistant acid phosphatase; TRAP-5b $=$ tartrate-resistant acid phosphatase-5b. 
Table 4. Animal studies.

\begin{tabular}{|c|c|c|c|c|c|c|c|}
\hline Reference & Location & Animal Model & Plum Product & Methods & Markers Measured & Duration & Results \\
\hline $\begin{array}{l}\text { Arjmandi et al. } \\
2010 \text { [15] }\end{array}$ & USA & $\begin{array}{l}\text { Sprague-Dawley } \\
\text { rats }\end{array}$ & $\begin{array}{l}\text { Dried plum } \\
\text { (P. domestica), } \\
\text { DP puree, DP juice, } \\
\text { DP pulp/skin, DPP }\end{array}$ & $\begin{array}{l}\text { After surgery to establish } \\
\text { bone loss, rats placed on } \\
\text { various diets } \\
\text { supplemented with } \\
13 \text { different combinations } \\
\text { of fructooligosaccharides } \\
\text { and DP vs. a control diet. }\end{array}$ & $\begin{array}{l}\text { BMD and BMC (whole } \\
\text { body, right femur, } \\
\text { 4th lumbar vertebrae), } \\
\text { calcium loss (4th lumbar), } \\
\text { TbS, serum OC, serum } \\
\text { IGF-1, calcium, phosphorus, } \\
\text { and magnesium. }\end{array}$ & 60 days & $\begin{array}{l}\text { Compared to the other treatments, diets } \\
\text { supplemented with } 5 \% \text { FOS and } 7.5 \% \text { DP was most } \\
\text { effective in reversing both right femur and fourth } \\
\text { lumbar BMD and fourth lumbar calcium loss while } \\
\text { significantly decreasing TbS. No significant effects of } \\
\text { treatment on serum or urine measures of } \\
\text { bone turnover. }\end{array}$ \\
\hline Bu et al. 2007 [16] & USA & $\begin{array}{l}\text { Male } \\
\text { Sprague-Dawley } \\
\text { rats }\end{array}$ & $\begin{array}{l}\text { Dried plum } \\
(P . \text { domestica }) \text { vs. } \\
\text { parathyroid } \\
\text { hormone }\end{array}$ & $\begin{array}{l}\text { Diet supplementation of } \\
\text { 6-month old male rats } \\
\text { with } 25 \% \text { DP vs. } \\
\text { a control diet. }\end{array}$ & $\begin{array}{l}\text { BMA, BMC, BMD (whole } \\
\text { body, femur, vertebrae), } \\
\text { trabecular architecture, } \\
\text { cortical bone, serum ALP, } \\
\text { serum protein, BV/TV, TbN, } \\
\text { TbSp, femur and vertebral } \\
\text { (connectivity density, SMI, } \\
\text { linear attenuation), total } \\
\text { force, stiffness, } \\
\text { physiological force. }\end{array}$ & 90 days & $\begin{array}{l}\text { DPs induced a significant increase in vertebra and } \\
\text { femoral BMD compared to controls. DPs induced a } \\
\text { significant increase in femur BMC compared to } \\
\text { controls. The DP group had significantly: } \\
\text { - Higher femur and vertebra BV/TV, TbN. } \\
\text { - Higher femur connectivity density, femur and } \\
\text { vertebral linear attenuation. } \\
\text { - Higher cortical thickness and cortical area. } \\
\text { - } \quad \text { Hower TbSp and femur SMI, } \\
\text { - } \quad \text { physher total force, stiffness, and } \\
\text { - Lower average von Mises stresses. }\end{array}$ \\
\hline $\begin{array}{l}\text { Deyhim et al. } \\
2005 \text { [17] }\end{array}$ & USA & $\begin{array}{l}\text { Sprague-Dawley } \\
\text { rats }\end{array}$ & $\begin{array}{l}\text { Dried plum } \\
\text { (P. domestica) }\end{array}$ & $\begin{array}{l}\text { Dietary supplementation } \\
\text { of adult osteopenic rats } \\
\text { with } 5 \%, 15 \% \text { or } 25 \% \text { DP } \\
\text { vs. a control diet. }\end{array}$ & $\begin{array}{l}\text { Serum ALP, TRAP activities, } \\
\text { calcium, magnesium, IGF-I, } \\
\text { BMD (femur, tibia, } \\
\text { vertebra), trabecular } \\
\text { microarchitecture, urinary } \\
\text { DPD, L4 BMD, BV/TV, } \\
\text { connectivity density, TbSp, } \\
\text { and TbTh. }\end{array}$ & 60 days & $\begin{array}{l}\text { Compared to OVX controls: } \\
\text { - } \quad \text { All DP groups had significantly higher femur } \\
\text { BMD, tibia BMD, as well as lower TbSp. } \\
\text { - } \quad 25 \% \text { DP groups had significantly higher L4 BMD, } \\
\text { BV/TV, and connectivity density. } \\
\text { - } \quad 15 \% \text { and } 25 \% \text { DP groups had significantly higher } \\
\text { TbN and lower TbTh. }\end{array}$ \\
\hline
\end{tabular}


Table 4. Cont

\begin{tabular}{|c|c|c|c|c|c|c|}
\hline Reference & Location & Animal Model & Plum Product & Methods & Markers Measured & Duration \\
\hline $\begin{array}{l}\text { Franklin et al. } \\
2006 \text { [18] }\end{array}$ & USA & $\begin{array}{l}\text { Male } \\
\text { Sprague-Dawley } \\
\text { rats }\end{array}$ & $\begin{array}{l}\text { Dried plum } \\
\text { (P. domestica) }\end{array}$ & $\begin{array}{l}\text { Dietary supplementation } \\
\text { of male rats with } 5 \% \text {, } \\
15 \% \text { or } 25 \% \text { DP vs. } \\
\text { a control diet. }\end{array}$ & $\begin{array}{l}\text { Whole body BMC, BMA, } \\
\text { BMD), BMC (femur, } \\
\text { L4 vertebra), trabecular } \\
\text { bone microarchitecture } \\
\text { markers (BV/TV, TbN, } \\
\text { TbSp), serum ALP, } \\
\text { osteocalcin, IGF-I, RANKL, } \\
\text { OPG, cortical strength, } \\
\text { cortical area, medullary } \\
\text { area, cortical porosity, distal } \\
\text { femur and L4 vertebral } \\
\text { (SMI, connectivity density, } \\
\text { LinAtt), IGF, DPD, } \\
\text { OPG, RANKL. }\end{array}$ & 90 days \\
\hline
\end{tabular}

$15 \%$ and $25 \%$ DP groups significantly prevented a

reduction in whole body BMD, as well as femur and

L4 vertebra BMC. $15 \%$ and $25 \%$ DP groups protected

against the decrease in mechanical strength required

to break the femur bone. Compared to controls:

- $5 \%$ and $25 \%$ DP groups had significantly higher

- $25 \%$ DP group had significantly higher L4 vertebra BV/TV, TbN and significantly lower L4 vertebra TbSp.

- All DP groups had significantly higher distal femur TbN and lower distal femur TbSp, DPD, RANKL.

- $15 \%$ and $25 \%$ DP groups had significantly higher cortical strength and lower vertebral SMI and OPG.

- $25 \%$ DP group had significantly lower femur SMI and higher femur and vertebral connectivity density, vertebral LinnAtt, and IGF.

- $5 \%$ and $25 \%$ DP groups had significantly higher LinAtt.

No significant differences on other markers measured.

Within both adult and old mice, increasing DP supplementation was associated with greater BV. Mice fed $25 \%$ DP had significantly greater BV compared to controls. Mice fed $25 \%$ DP had significantly greater

$\mathrm{BV} / \mathrm{TV}, \mathrm{TbN}, \mathrm{TbSp}, \mathrm{P} 1 \mathrm{NP}$ SMI, connective density,

$\begin{array}{llll}\begin{array}{l}\text { Halloran et al. } \\ 2010[19]\end{array} & \text { USA } & \begin{array}{l}\text { Harlan Sprague } \\ \text { Dawley mice }\end{array} & \begin{array}{l}\text { Dried plum } \\ \text { (P. domestica) }\end{array}\end{array}$

Dietary supplementation of adult and old male vs. a control diet. 年, 6 months Medullary area, BMD and PYD magnitude of the percent changes between control mice and those fed $25 \%$ DP were significantly greater in adult vs. old mice. Compared to controls:

- Adult mice fed 25\%DP had higher BV/TV, TbN

connective density, and lower SMI.

- Old mice fed $25 \%$ DP had higher degree of anisotropy and cortical thich

- Old mice fed $15 \%$ DP had higher cortical area.

No significant differences on other markers measured. 
Table 4. Cont.

\begin{tabular}{|c|c|c|c|c|c|c|c|}
\hline Reference & Location & Animal Model & Plum Product & Methods & Markers Measured & Duration & Results \\
\hline $\begin{array}{l}\text { Johnson et al. } \\
2011[20]\end{array}$ & USA & $\begin{array}{l}\text { Sprague-Dawley } \\
\text { rats }\end{array}$ & $\begin{array}{l}\text { Dried plum } \\
\text { (P. domestica) }\end{array}$ & $\begin{array}{l}\text { Female ovarian hormone } \\
\text { deficient rats a fed control, } \\
\text { soy, soy + FOS, soy + 7.5\% } \\
\text { DP, and soy + } 7.5 \% \text { DP + } \\
\text { FOS diet vs. a control diet. }\end{array}$ & $\begin{array}{l}\text { BMD, BMC (Whole body, } \\
\text { right femur, 4th lumbar } \\
\text { vertebrae), serum ALP, } \\
\text { urinary creatinine, urinary } \\
\text { DPD, femur strength, TbN, } \\
\text { BV/TV, TbTh, and TbSp. }\end{array}$ & 60 days & $\begin{array}{l}\text { Whole body and 4th lumbar BMD were significantly } \\
\text { higher in diets with DP + FOS compared to the } \\
\text { control and soy diets. No significant differences on } \\
\text { other markers measured. }\end{array}$ \\
\hline $\begin{array}{l}\text { Leotoing et al. } \\
2016 \text { [21] }\end{array}$ & France & Wistar rats & $\begin{array}{l}\text { High and low } \\
\text { chlorogenic } \\
\text { acid dried plum } \\
\text { (P. domestica) and } \\
\text { DP juice } \\
\text { concentrate }(15 \%)\end{array}$ & $\begin{array}{l}\text { Female rats High and low } \\
\text { chlorogenic acid dried } \\
\text { plum (P. domestica) and } \\
\text { DP juice concentrate (15\%) } \\
\text { diets vs. a control diet. }\end{array}$ & $\begin{array}{l}\text { Urinary DPD, OC, CPII, } \\
\text { CTX-II, BMD (Total femoral, } \\
\text { metaphyseal), BMC, urine } \\
\text { calcium, primary } \\
\text { pre-osteoblasts } \\
\text { (proliferation, ALP), bone } \\
\text { remodeling index, and } \\
\text { cartilage remodeling index. }\end{array}$ & $\begin{array}{l}90 \text { days } \\
\text { (in vivo), } \\
7 \text { days } \\
\text { (ex vivo) }\end{array}$ & $\begin{array}{l}10 \text { and } 50 \mu \mathrm{mol} / \mathrm{L} \text { concentrations of neochlorogenic, } \\
\text { chlorogenic, or caffeic acid significantly decreased } \\
\text { pre-osteoblast ALP activity and increased } \\
\text { pre-osteoblast proliferation. The low chlorogenic acid } \\
\text { DP juice and DP juice concentrate groups showed } \\
\text { significantly higher trabecular distal BMD, } \\
\text { significantly increased cortical BMD, and increased } \\
\text { total BMC compared to control. High chlorogenic acid } \\
\text { DP juice group had significantly higher trabecular } \\
\text { distal BMD compared to controls. High chlorogenic } \\
\text { acid DP juice, low chlorogenic acid DP juice + fiber } \\
\text { and low chlorogenic acid DP juice concentrate } \\
\text { significantly prevented increase in OC. } \\
\text { Low chlorogenic acid DP juice + fiber and low } \\
\text { chlorogenic acid DP juice concentrate significantly } \\
\text { prevented increase in DPD. Both high and low } \\
\text { chlorogenic acid DP juice and DP juice concentrate } \\
\text { lead to higher urinary calcium excretion compared to } \\
\text { controls. Only high chlorogenic acid DP juice } \\
\text { significantly counteracted the decrease in CPII. } \\
\text { Only the high chlorogenic acid DP juice group had } \\
\text { significantly higher CRI. }\end{array}$ \\
\hline $\begin{array}{l}\text { Monsefi et al. } \\
2013 \text { [22] }\end{array}$ & Iran & Pregnant mice & $\begin{array}{l}\text { Dried plum } \\
\text { (P. domestica) extract } \\
(8 \mathrm{~mL} / \mathrm{kg}) \text { and DP } \\
\text { hydroalcoholic } \\
\text { extracts }(1.6 \mathrm{~g} / \mathrm{kg})\end{array}$ & $\begin{array}{l}\text { Pregnant mice were fed } \\
\text { DP extracts vs. a control } \\
\text { diet and outcomes } \\
\text { measured on their fetuses. }\end{array}$ & $\begin{array}{l}\text { Serum calcium, magnesium, } \\
\text { ALP, bone calcium, and } \\
\text { phosphorus. }\end{array}$ & 30 days & $\begin{array}{l}\text { Non-pregnant mice fed DP extract had significantly } \\
\text { higher bone calcium compared to non-pregnant } \\
\text { controls. Non-pregnant mice fed DP hydroalcoholic } \\
\text { extracts had significantly higher bone phosphorus } \\
\text { compared to non-pregnant controls. Non-pregnant } \\
\text { mice fed both DP extract and DP hydroalcoholic } \\
\text { extract had significantly higher bone calcium } \\
\text { compared to non-pregnant controls. }\end{array}$ \\
\hline
\end{tabular}


Table 4. Cont.

\begin{tabular}{|c|c|c|c|c|c|c|c|}
\hline Reference & Location & Animal Model & Plum Product & Methods & Markers Measured & Duration & Results \\
\hline $\begin{array}{l}\text { Pawlowski et al. } \\
2014 \text { [23] }\end{array}$ & USA & $\begin{array}{l}\text { Sprague-Dawley } \\
\text { rats }\end{array}$ & $\begin{array}{l}\text { Dried plum powder } \\
\text { extract }(0.20 \% \text { and } \\
0.45 \% w / w \text { total } \\
\text { dietary polyphenols })\end{array}$ & $\begin{array}{l}\text { Randomized, crossover } \\
\text { intervention trial to } \\
\text { evaluate } 12 \text { different } \\
\text { polyphenolics containing } \\
\text { diets on bone turnover. }\end{array}$ & $\begin{array}{l}\text { Urine calcium (total and } \\
{ }^{45} \mathrm{Ca} \text { ), NTx and ALP. }\end{array}$ & 10 days & $\begin{array}{l}\text { Bone calcium retention was significantly improved } \\
\text { due to dietary intervention with } 0.45 \% \text { DP extract } \\
\text { compared to baseline. } 0.45 \% \text { DP extract improved } \\
\text { bone calcium retention compared with the } 0.20 \% \text { DP } \\
\text { extract. No significant effect on other outcomes. }\end{array}$ \\
\hline $\begin{array}{l}\text { Rendina et al. } \\
2012 \text { [24] }\end{array}$ & USA & $\begin{array}{l}\text { Adult female } \\
\text { C57BL/6J mice }\end{array}$ & $\begin{array}{l}\text { Dried plum } \\
\text { (Prunus domestica) }\end{array}$ & $\begin{array}{l}\text { Adult female mice placed } \\
\text { on } 5 \%, 15 \% \text { or } 25 \% \text { DP } \\
\text { intervention vs. } \\
\text { a control diet. }\end{array}$ & $\begin{array}{l}\text { BMA, BMC and BMD of the } \\
\text { 4th to 5th lumbar vertebrae } \\
\text { (L4-L5), TbN, BV/TV, TbTh, } \\
\text { TbSp, connectivity density, } \\
\text { SMI, PINP IGF-I, NFATc, } \\
\text { Runx2, biomechanical } \\
\text { properties of trabecular } \\
\text { bone, OC, IL- } 6 \text {, and TNF- } \alpha \text {. }\end{array}$ & 4 weeks & $\begin{array}{l}\text { Mean BMC and BMA were significantly higher in the } \\
25 \% \text { DP group compared to the control. 15\% DP } \\
\text { group had a significantly higher plasma IGF-1 } \\
\text { compared to the control. } 15 \% \text { and } 25 \% \text { DP groups } \\
\text { significantly increased BV/TV compared to the } \\
\text { control. } 15 \% \text { and } 25 \% \text { DP groups significantly } \\
\text { decreased TbSp beyond that of the control group. } 15 \% \\
\text { and } 25 \% \text { DP groups experienced a significant increase } \\
\text { in vertebra TbTh compared to the control. } 15 \% \text { and } \\
25 \% \text { DP groups had significantly lower Von Mises } \\
\text { stress distribution compared to the control. } 15 \% \text { and } \\
25 \% \text { DP groups had significantly higher vertebral } \\
\text { connective density and tibia apparent mean/density, } \\
\text { and lower vertebral SMI and OC expression and } \\
\text { TNF- } \alpha \text {. } 25 \% \text { DP group had significantly higher } \\
\text { apparent mean/density and tibia connective density, } \\
\text { and significantly lower tibia SMI. } 25 \% \text { DP group } \\
\text { significantly increased TbN compared to the control. } \\
\text { All doses of DP groups had significantly lower plasma } \\
\text { PINP, NFATc and Runx2 compared to the control. }\end{array}$ \\
\hline
\end{tabular}


Table 4. Cont.

\begin{tabular}{|c|c|c|c|c|c|c|c|}
\hline Reference & Location & Animal Model & Plum Product & Methods & Markers Measured & Duration & Results \\
\hline $\begin{array}{l}\text { Rendina et al. } \\
2013 \text { [25] }\end{array}$ & USA & $\begin{array}{l}\text { Adult osteopenic } \\
\text { ovariectomized } \\
\text { C57BL/ } 6 \text { mice }\end{array}$ & $\begin{array}{l}\text { Dried plum } \\
\text { (Prunus domestica), } \\
25 \%\end{array}$ & $\begin{array}{l}\text { This study was designed } \\
\text { to compare the efficacy of } \\
\text { DP, apple, apricot, grape, } \\
\text { and mango vs. a control } \\
\text { in the restoration of bone } \\
\text { in an osteopenic mouse } \\
\text { model. }\end{array}$ & $\begin{array}{l}\text { Whole body and L4-5 } \\
\text { (BMA, BMC, BMD), TbN, } \\
\text { BV/TV, TbTh, TbSp, SMI, } \\
\text { biomechanical testing of } \\
\text { vertebra and tibia, } \\
\text { connective density, NFATc1, } \\
\text { ALP, Col1a1, OC, Bak1, } \\
\text { Casp3, and Casp9. }\end{array}$ & 8 weeks & $\begin{array}{l}\text { Compared to the control the DP group had } \\
\text { significantly higher whole body and spine BMA, } \\
\text { BMD and BMC. DP group had significantly higher } \\
\text { vertebral BV/TV, TbN, TbTh, connective density, SMI, } \\
\text { and trabecular density compared to the control group. } \\
\text { DP group had significantly higher proximal tibia } \\
\text { BV/TV compared to the control group. DP group had } \\
\text { significantly higher vertebral total force, stiffness, size } \\
\text { independent stiffness compared to the control group. } \\
\text { DP group had significantly lower NFATc1 compared } \\
\text { to the control group. DP group had significantly } \\
\text { higher Bak1 and lower Casp3 and compared to the } \\
\text { control group. No significant differences on } \\
\text { other markers. }\end{array}$ \\
\hline $\begin{array}{l}\text { Schreurs et al. } \\
2016 \text { [26] }\end{array}$ & USA & $\begin{array}{l}\text { Male } \\
\text { C57BL/6J mice }\end{array}$ & $\begin{array}{l}\text { Dried plum } \\
\text { (Prunus domestica), } \\
25 \%\end{array}$ & $\begin{array}{l}\text { This study randomized } \\
\text { mice to } 25 \% \text { DP } \\
\text { intervention vs. a control } \\
\text { to protect from bone loss } \\
\text { and then later exposed } \\
\text { them to ionizing } \\
\text { radiation. }\end{array}$ & $\begin{array}{l}\text { Nfe2l2, RANL, MCP-1, } \\
\text { OPG, TNF- } \alpha, \text { TbN, BV/TV, } \\
\text { TbTh, TbSp, }\end{array}$ & $7-21$ days & $\begin{array}{l}\text { Compared to the irradiated controls, levels of Nfe2l2, } \\
\text { RANKL, MCP-1, OPG, and TNF- } \alpha \text { in the DP group } \\
\text { were not statistically different. After exposure to } \\
\text { radiation, DP mice did not have any significant } \\
\text { decrease in TbN, BV/TV, TbTh or TbSp indicating a } \\
\text { radio-protective effects against cancellous bone loss } \\
\text { compared to irradiated controls. DP fed mice had } \\
\text { significantly higher BV/TV, TbTh and TbN after being } \\
\text { exposed to simulated space radiation compared to } \\
\text { control diet. }\end{array}$ \\
\hline
\end{tabular}


Table 4. Cont.

\begin{tabular}{|c|c|c|c|c|c|c|c|}
\hline Reference & Location & Animal Model & Plum Product & Methods & Markers Measured & Duration & Results \\
\hline & & & & \multirow{3}{*}{$\begin{array}{l}\text { Skeletally mature } \\
\text { (6-month-old) and } \\
\text { growing (1- and } \\
\text { 2-month-old) male mice } \\
\text { were placed on a } 5 \%, 15 \% \\
\text { or 25\% DP intervention vs. } \\
\text { a control diet. }\end{array}$} & \multirow{3}{*}{$\begin{array}{l}\text { BV/TV, TbTh, TbN, SMI, } \\
\text { OcS, ObS, MAR, MS/BS, } \\
\text { BFR/BS, Ctsk, OPG, } \\
\text { RANKL, CTX, and P1NP. }\end{array}$} & \multirow{3}{*}{$1-4$ weeks } & $\begin{array}{l}\mathrm{BV} / \mathrm{TV} \text { and TbTh significantly increased and SMI } \\
\text { significantly decreased after } 2 \text { and } 4 \text { weeks of DP. TbN } \\
\text { significantly increased after } 4 \text { weeks of DP. } \\
\text { After } 2 \text { and } 4 \text { weeks of DP: } \\
\text { - OcS, ObS, MAR, MS/BS, BFR/BS } \\
\text { decreased significantly. } \\
\text { - Osteoclasts significantly decreased. }\end{array}$ \\
\hline $\begin{array}{l}\text { Shahnazari et al. } \\
2016 \text { [27] }\end{array}$ & USA & C57Bl/6 mice & $\begin{array}{l}\text { Dried plum } \\
\text { (Prunus domestica) }\end{array}$ & & & & $\begin{array}{l}\text { DP fed mice had significantly lower: } \\
\text { - Ctsk gene expression. } \\
\text { - Immune-related cytokines (IL-1a, IL-1b, IL-10, } \\
\text { IL-12, IL-13, IL-17, TNF- } \alpha \text {, and MCP-1). } \\
\text { - CTX }\end{array}$ \\
\hline & & & & & & & $\begin{array}{l}\text { BV/TV increased significantly in mice fed } 5 \%, 15 \% \text { or } \\
25 \% \text { DP. TbTh increased significantly among mice fed } \\
25 \% \text { DP. TbN increased significantly in mice fed } 5 \% \text {, } \\
15 \% \text { or } 25 \% \text { DP. SMI decreased significantly for mice } \\
\text { fed } 15 \% \text { and } 25 \% \text { DP. Among } 2 \text {-month-old mice, ObS } \\
\text { increased significantly among mice fed } 5 \%, 15 \% \text { or } \\
25 \% \text { DP. Among 3-month-old mice, ObS increased } \\
\text { significantly among mice fed } 5 \% \text { DP. No significant } \\
\text { differences on other markers. }\end{array}$ \\
\hline
\end{tabular}


Table 4. Cont.

\begin{tabular}{|c|c|c|c|c|c|c|c|}
\hline Reference & Location & Animal Model & Plum Product & Methods & Markers Measured & Duration & Results \\
\hline \multirow{12}{*}{$\begin{array}{l}\text { Smith et al. } \\
2014 b[28]\end{array}$} & \multirow{12}{*}{ USA } & \multirow{12}{*}{$\begin{array}{l}\text { Female } \\
\text { Sprague-Dawley } \\
\text { rats }\end{array}$} & \multirow{12}{*}{$\begin{array}{l}\text { Dried plum } \\
\text { (Prunus domestica) }\end{array}$} & \multirow{12}{*}{$\begin{array}{l}\text { Osteopenic rats were } \\
\text { placed on } 5 \%, 15 \% \text { or } 25 \% \\
\text { DP intervention vs. } \\
\text { a control diet. }\end{array}$} & \multirow{12}{*}{$\begin{array}{l}\text { BMD (whole body, femur } \\
\text { and vertebra), BV/TV, TbN, } \\
\text { TbSp, connective density, } \\
\text { TbTh (proximal tibia, } \\
\text { vertebra), Cortical thickness, } \\
\text { cortical area, medullary } \\
\text { area, cortical porosity, DPD, } \\
\text { P1NP, cancellous BFR and } \\
\text { MS/BS, MAR, MS/bone } \\
\text { area, BFR/BV, Periosteal } \\
\text { (BFR, MS, MAR), } \\
\text { endocortical (BFR, MS, } \\
\text { MAR), Bmp2, Bmp4, Coll1a, } \\
\text { IGF-1, Nfatc1, and RANKL. }\end{array}$} & \multirow{12}{*}{6 weeks } & Compared to controls: \\
\hline & & & & & & & $\begin{array}{l}\text { - Vertebral BMD increased significantly in } 15 \% \\
\text { and } 25 \% \text { DP groups } \\
\text { - Femur BMD increased significantly in } 5 \%, 15 \% \text {, } \\
\text { and } 25 \% \text { DP groups } \\
\text { - Whole body BMD increased significantly in } 5 \% \text {, } \\
15 \% \text {, and } 25 \% \text { DP groups }\end{array}$ \\
\hline & & & & & & & $\begin{array}{l}\text { Within the vertebra and when compared to controls: } \\
\text { - BV/TV, TbN, TbSp, connective density increased } \\
\text { significantly in 5\%, } 15 \% \text { and } 25 \% \text { DP groups. } \\
\text { - TbTh increased significantly in } 15 \% \text { and } 25 \% \\
\text { DP groups. }\end{array}$ \\
\hline & & & & & & & $\begin{array}{l}\text { Within the proximal tibia and when compared to } \\
\text { controls: TbSp decreased significantly in } 15 \% \text { and } 25 \% \\
\text { DP groups. When compared to controls: cortical } \\
\text { thickness increased significantly in 5\%, } 15 \% \text {, and } 25 \% \\
\text { DP groups. } 25 \% \text { of DP significantly suppressed } \\
\text { increase in urinary DPD excretion. 5\%, } 5 \% \text {, and 25\% } \\
\text { DP groups significantly suppressed serum } \\
\text { P1NP.Compared to controls: }\end{array}$ \\
\hline & & & & & & & $\begin{array}{l}\text { - } 15 \% \text { and } 25 \% \text { DP groups significantly } \\
\text { suppressed increase in cancellous BFR and } \\
\text { MS/BS. }\end{array}$ \\
\hline & & & & & & & $\begin{array}{l}\text { - } 15 \% \text { and } 25 \% \text { DP groups significantly } \\
\text { suppressed increase in cancellous BFR and } \\
\text { MS / BS. }\end{array}$ \\
\hline & & & & & & & \\
\hline & & & & & & & $\begin{array}{l}\text { - MS/bone area, BFR/BV, Periosteal BFR levels } \\
\text { were significantly decreased in 5\%,15\%, and } \\
25 \% \text { DP groups. }\end{array}$ \\
\hline & & & & & & & $\begin{array}{l}\text { All groups of DP increased significantly } \\
\text { Bmp4 expression. }\end{array}$ \\
\hline & & & & & & & $\begin{array}{l}\text { - } 25 \% \text { group significantly increased } \\
\text { IGF-1 expression. }\end{array}$ \\
\hline & & & & & & & $\begin{array}{l}\text { - Relative abundance of NFATc1 mRNA was } \\
\text { significantly lower in all DP groups. }\end{array}$ \\
\hline & & & & & & & No significant differences on other markers. \\
\hline
\end{tabular}


Table 4. Cont.

\begin{tabular}{|c|c|c|c|c|c|c|c|}
\hline Reference & Location & Animal Model & Plum Product & Methods & Markers Measured & Duration & Results \\
\hline $\begin{array}{l}\text { Smith et al. } \\
\text { 2014a [29] }\end{array}$ & USA & $\begin{array}{l}\text { Male } \\
\text { C57BL/6 mice }\end{array}$ & $\begin{array}{l}\text { Dried plum } \\
\text { (Prunus domestica) }\end{array}$ & $\begin{array}{l}\text { Osteopenic rats were } \\
\text { placed on } 25 \% \text { DP } \\
\text { intervention vs. } \\
\text { a control diet. }\end{array}$ & $\begin{array}{l}\text { Whole-body and vertebral } \\
\text { (BMD, BMC, BMA) lumbar } \\
\text { vertebra, distal femur, } \\
\text { femur mid-diaphysis } \\
\text { (BV/TV, TbN, TbTh, TbS, } \\
\text { connective density, SMI), } \\
\text { P1NP, PYD, glutathione } \\
\text { peroxidase activity, OcS, } \\
\text { ObS, MS, BFR, MAR, Pparc, } \\
\text { Osx, Bmp2, Bmp4, ALP, } \\
\text { Col1a1, OC, RANKL, OPG, } \\
\text { NFATc1, and Ctsk. }\end{array}$ & 4 or 12 weeks & $\begin{array}{l}\text { At 4weeks: } \\
\text { - } \quad \text { Whole body BMD, vertebra (BMD, BMC) were } \\
\text { - } \quad \text { significantly higher in the DP group. } \\
\text { corticar BV/ thickness, were significantly higher in } \\
\text { the DP group. } \\
\text { - SMI was significantly lower in the DP group. } \\
\text { At } 12 \text { weeks: } \\
\text { Whole body BMA, BMC and BMD, as well as } \\
\text { vertebra BMD and BMC were significantly } \\
\text { higher in the DP group. } \\
\text { BV/TV (lumbar and distal femur), trabecular } \\
\text { number (lumbar and distal femur), connectivity } \\
\text { density (lumbar and distal femur), and femur } \\
\text { cortical thickness, were significantly higher in } \\
\text { the DP group. } \\
\text { Trabecular separation (lumbar and distal femur), } \\
\text { SMI (lumbar and distal femur) were significantly } \\
\text { lower in the DP group. At } 4 \text { and } 12 \text { weeks, } \\
\text { serum P1NP of DP group were significantly } \\
\text { reduced compared to controls. At 12 weeks, } \\
\text { serum PYD was significantly lower in the DP } \\
\text { group. The DP group had significantly higher } \\
\text { glutathione peroxidase activity than the control } \\
\text { at } 12 \text { weeks. At } 4 \text { weeks, the DP group had } \\
\text { significantly lower OcS, ObS, MS, and BMR. } \\
\text { At } 4 \text { weeks, the DP group had significantly } \\
\text { higher Pparc; lower Osx, Bmp4, ALP, Colla1, } \\
\text { Bglap2, RANKL, and NFATc1. }\end{array}$ \\
\hline
\end{tabular}


Table 4. Cont.

\begin{tabular}{|c|c|c|c|c|c|c|c|}
\hline Reference & Location & Animal Model & Plum Product & Methods & Markers Measured & Duration & Results \\
\hline $\begin{array}{l}\text { Arjmandi et al. } \\
2001[30]\end{array}$ & USA & $\begin{array}{l}\text { Female } \\
\text { Sprague-Dawley } \\
\text { rats }\end{array}$ & (Prunus domestica) & $\begin{array}{l}\text { Female rats were either } \\
\text { ovariectomized or sham } \\
\text { operated. The } \\
\text { ovariectomized groups } \\
\text { were then fed either a } 5 \% \\
\text { or } 25 \% \text { DP supplemented } \\
\text { diet vs. a control diet. }\end{array}$ & $\begin{array}{l}\text { Trabecular (total area, bone } \\
\text { area, \% bone area). Cortical } \\
\text { (total area, bone area, } \\
\text { marrow space, endosteal } \\
\text { perimeter, and periosteal } \\
\text { perimeter. }\end{array}$ & 45 days & $\begin{array}{l}\text { Compared to the controls the } 25 \% \text { DP group had } \\
\text { significantly higher trabecular BA. Unreported results } \\
\text { (data not shown): } \\
\text { - } \quad \text { DP diets dose-dependently enhanced IGF-1. }\end{array}$ \\
\hline
\end{tabular}

ALP = alkaline phosphatase; BAK1 = BRI1-associated kinase 1; BFR = bone formation rate; BMA = bone mineral area; $\mathrm{BMC}=$ bone mineral content; $\mathrm{BMD}=$ bone mineral density; BMP2 = bone morphogenetic protein-2; BMP4 = bone morphogenetic protein-4; BS = bone surface; BS = bone surface; BV = bone volume; Casp3 = caspase-3; Casp9 = caspase-9; Col1a1 = collagen type 1a1; Coll1a = collagen type 1; CPII = C-propeptide of type II collagen; Ctsk = cathepsin K; CTX = C-terminal telopeptide of type II collagen; DP = dried plum; $\mathrm{DPD}=$ deoxypyridinoline; DPP = dried plum polyphenols; FOS = fructooligosaccharides; IGF = insulin-like growth factor; IGF-1 = Insulin-like growth factor-1; IL-6 = Interlukin-6; LinAtt = Linear X-ray attenuation coefficient; MAR = Mineral absorption rate; MCP-1 = Monocyte chemoattractant 1 ; MS = Mineralizing surface; NFATc = Nuclear factor of activated T cells; NFATc1 = Nuclear factor of activated T cells-1; Nfe212 = Nuclear factor erythroid derived 212; NTx = N-telopeptides of type-1 collagen; ObS = Osteoblast surface; OC = osteocalcin; OcS = Osteoclast surface; OPG = osteoprotegerin; Osx = osterix; PINP = procollagen type I N-terminal propeptide; Pparc = proliferator-activated receptor gamma; PYD = pyridinoline; $\mathrm{RANKL}=$ receptor activator of nuclear factor kappa-B ligand; Runx2 $=$ Runt-related protein 2; $\mathrm{SMI}=$ structural model indexTbN $=$ trabecular bone number; TbSp $=$ trabecular bone separation; $\mathrm{TbTh}=$ trabecular thickness; TNF-a $=$ tumor necrosis factor-alpha; TRAP = tartrate-resistant acid phosphatase; $\mathrm{TV}=$ trabecular volume. 
Table 5. Cell studies.

\begin{tabular}{|c|c|c|c|c|c|c|c|}
\hline Reference & Location & Cell Type & Plum Product & Dose & Methods & Markers Measured & Results \\
\hline Bu et al. 2008 [31] & USA & $\begin{array}{l}\text { RAW } 264.7 \text { murine } \\
\text { macrophage cells }\end{array}$ & DPE (P. domestica) & $\begin{array}{l}0,10,20, \\
\text { or } 30 \mu \mathrm{g} / \mathrm{mL} \text { dried } \\
\text { plum polyphenols }\end{array}$ & $\begin{array}{l}\text { Cells were cultured } \\
\text { and treated with } \\
\text { various doses of } \\
\text { dried plum extract. }\end{array}$ & $\begin{array}{l}\text { Osteoclast } \\
\text { differentiation and } \\
\text { activity. }\end{array}$ & $\begin{array}{l}\text { DPE suppressed osteoclast } \\
\text { differentiation and activity under } \\
\text { normal, oxidative stress, } \\
\text { and inflammatory conditions. }\end{array}$ \\
\hline Bu et al. 2009 [32] & USA & MC3T3-E1 cells & DPP (P. domestica) & $\begin{array}{l}0,2.5,5,10 \text { and } \\
20 \mu \mathrm{g} / \mathrm{mL}\end{array}$ & $\begin{array}{l}\text { Cells were plated } \\
\text { and pretreated with } \\
\text { dried plum extracts } \\
\text { and later stimulated } \\
\text { with TNF- } \alpha \text {. }\end{array}$ & $\begin{array}{l}\text { Osteoblast function, } \\
\text { mineralized nodule } \\
\text { formation, and ALP. }\end{array}$ & $\begin{array}{l}\text { DPP significantly increased } \\
\text { intracellular ALP activity under } \\
\text { normal conditions and significantly } \\
\text { restored the TNF- } \alpha \text {-induced } \\
\text { suppression of intracellular ALP } \\
\text { activity. DPP increased mineralized } \\
\text { nodule formation under normal and } \\
\text { inflammatory conditions. } \\
\text { DPP increased osteoblast activity and } \\
\text { function. }\end{array}$ \\
\hline $\begin{array}{l}\text { Hooshmand et al. } \\
2015 \text { [33] }\end{array}$ & USA & RAW 264.7 cells & $\begin{array}{l}\text { DPP } \\
\text { (Prunus domestica L.) }\end{array}$ & $\begin{array}{l}0,0.1,1,10,100 \\
1000 \mu \mathrm{g} / \mathrm{mL} \text { DPP }\end{array}$ & $\begin{array}{l}\text { Cells were treated to } \\
\text { different doses } \\
\text { of DPP. }\end{array}$ & $\mathrm{NO}, \mathrm{COX}-2$, and $\mathrm{MA}$ & 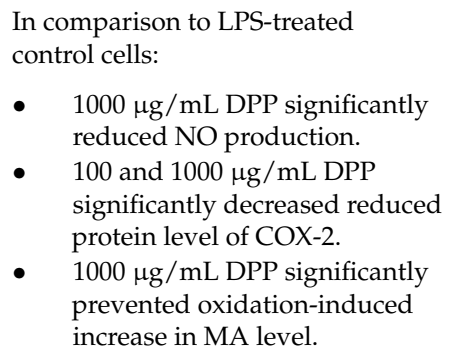 \\
\hline
\end{tabular}

ALP = alkaline phosphatase; COX-2 = cyclooxygenase-1; DPE = dried plum extract; DPP = dried plum polyphenols; $\mathrm{MA}=$ malondialdehyde; NO = nitric oxide; TNF-a = tumor necrosis factor-alpha. 


\section{Results}

The literature search of the PubMed database yielded 50 articles [10-61]. After title/abstract review, 22 articles were screened in full-text and included in this comprehensive review [10-29,31,32]. Two additional studies $[30,33]$ were included after examination of the reference lists of the 22 studies identified in the literature search. Data and results from each of the 24 included studies are listed in Tables 3-5.

Four clinical trials were identified in this comprehensive review [10-14]. Most the studies retrieved for full-text review were animal studies involving either rats or mice (16 total) [15-30], although three (cell) studies were also identified [31-33]. No observational studies were identified in the PubMed literature search or after examination of the reference lists of included studies.

\section{Discussion}

Dried plums are being increasingly recognized for their role in bone health. This comprehensive review supports that consumption of dried plums is safe and may be a bone healthy option for postmenopausal women wishing to satisfy daily requirement for fruit as outlined by the 2015-2020 DGA. It is important to note that dried plums contain a higher amount of vitamin K as compared to other commonly-consumed fruits, which may influence bone health by helping to improve calcium balance.

The quality of clinical studies included in this comprehensive review was acceptable, noting that none utilized a sample size based on a priori power calculation, nor were the treatment allocations able to be concealed from the participants and/or investigators. All four clinical studies identified were derived from the same laboratory group [10-14], meriting the need for replication by additional investigators. Clinical studies included in this review had several limitations, such as a short duration of 3-12 months, which is a narrow window to see significant changes in BMD. All four clinical studies were un-blinded, and only the Hooshmand et al. 2016 [11] had an inactive placebo group. The Hooshmand et al. 2011 [10] and Hooshmand et al. 2014 [13] manuscripts used dried apples as the control group and represent the same study population with additional biomarkers being measured for the latter publication post hoc (BMD data are presented twice). Participants in this study also received $500 \mathrm{mg}$ of calcium and $400 \mathrm{IU}$ of vitamin D during the intervention [10,13], even though administration of these supplements was equal across both arms. Simonavice et al., 2014 [14], assessed the effects of dried plums and resistance training vs. resistance training alone on blood markers of bone and inflammation in female breast cancer survivors. While this study found null effects, these results are likely not generalizable to healthy postmenopausal women experiencing normal hormone-related bone loss. Resistance training has been shown to have a larger effect on preventing bone loss as compared to most dietary interventions and could have masked the much smaller the effects, if present, exerted by dried plums. The Hooshmand et al., 2016 [11], found that dried plum consumption at 50-100 g/day for a period of six months prevented loss of total body BMD, but not spine, hip or ulna BMD, likely due to its shorter duration.

Consistent improvements in BMD at several sites were noted in animal studies designed to model conditions at or before peak bone mass, pregnancy, post-menopause, osteopenia and/or osteoporosis. Rat models of ovarian hormone deficiency have been used for over 25 years to simulate postmenopausal bone loss in humans. Ovarian hormone deficient rats and postmenopausal women have many similar characteristics when it comes to bone loss. These characteristics include increased rates of bone turnover with resorption exceeding formation, an initial rapid phase of bone loss followed by a slower phase due to the ovariectomy, greater loss of trabecular vs. cortical bone, decreased intestinal absorption of calcium and a similar response to drug (e.g., bisphosphonate therapy) and lifestyle interventions (e.g., physical activity) [41]. Indeed, animal studies show that dried plums and/or their polyphenol-rich extracts can beneficially affect both BMD and bone biomarkers. The animal and cell studies presented in this comprehensive review are consistent with and supportive of the theory that a diet high in phenolics and/or flavonoids may enhance bone formation and 
inhibit bone resorption through their actions on cell signaling pathways that influence osteoblast and osteoclast differentiation [62]. Total body BMD and BMD at specific sites, as well as several blood biomarkers, including AP, BAP, BSAP, OPG, RANKL and TRAP-5b, have been shown to be consistently and beneficially impacted across both clinical and animal studies. Animal studies also collectively support that dried plums may beneficially influence bone area and micro-architecture.

Several bone turnover markers seemed to be improved among clinical studies; however, there was a lack of consistency among many of the markers across and between both clinical and animal studies. For instance, Arjmandi et al., 2002 [12], found significant increases in bone alkaline phosphatase (BAP), but the latter larger study Hooshmand et al., 2011 [10], reported a decrease in BAP. Noting the abundance of bone turnover markers measured in both research and the clinical setting, the International Osteoporosis Foundation (IOF) and International Federation of Clinical Chemistry and Laboratory Medicine (IFCC) Bone Markers Working group recently reviewed the scientific literature to determine the clinical potential of bone turnover markers, which includes the prediction of fracture risk and monitoring treatments for osteoporosis [63]. The IOF/IFCC working group identified one bone resorption marker (s-CTX, serum C-terminal telopeptide of type I collagen) and one bone formation marker (s-PINP, serum procollagen type $1 \mathrm{~N}$ propeptide) to be used as reference markers and measured by standardized assays in observational and intervention studies [63]. While only one animal study assessed the effects of dried plums on CTX [27] and two on PINP [27,28], collectively, the animal studies included in this review showed beneficial effects of dried plums and/or their polyphenol-rich extracts on most, but not all, markers of bone turnover. Nevertheless, bone turnover markers are likely too premature in their standardization and clinical utility to accurately predict small changes in bone, as expected in dietary interventions. Differences in study design, dose and duration may also contribute to the inconsistencies in the bone turnover markers measured across and between rodent and cell studies.

\section{Future Research}

Identification of the active components, particularly individual phenolics, and their potential modes of action are necessary to fully understand the overall effect of dried plums on bone health across the lifespan. While existing data indicate that consumption of dried plums may be beneficial in postmenopausal women with ongoing bone loss, future clinical and prospective cohort studies in premenopausal women, men and adolescents prior to peak bone mass accrual are necessary to confirm their effects across the population and to make generalizable dietary guidance statements.

Recent epidemiological studies show that phenolic compounds may have a stronger association with bone than general fruit and vegetable consumption [62]. Even though BMD is a validated biomarker of bone health, fractures represent the most significant clinical endpoint of bone health across the lifespan. Prospective cohort studies designed to assess potential associations of dried plum intake on both fracture risk and changes in BMD across the population and various subpopulations are greatly needed to confirm the findings of studies included in this comprehensive review.

\section{Conclusions}

Dried plums are an easy means to help individuals meet their daily recommendations for fruit intake. The beneficial effects of dried plums on bone health may be in part due to the unique variety of phenolics and nutrients present in the fruit. Animal and cell studies suggest that dried plums and/or their extracts enhance bone formation and inhibit bone resorption through their actions on cell signaling pathways that influence osteoblast and osteoclast differentiation; however, results on specific markers are not consistent across and between studies. Animal studies are somewhat consistent with small clinical interventions that show dried plums may exert beneficial effects on total body and site-specific BMD. Long-term prospective cohort studies using fractures and BMD as primary endpoints are needed to confirm the effects of smaller clinical, animal and mechanistic studies. No adverse effects were noted among any of the studies included in this comprehensive review. While the data are not completely 
consistent, this review suggests that postmenopausal women may safely consume dried plums as part of their fruit intake recommendations given their potential to have protective effects on bone loss.

Acknowledgments: Funding for the development of the manuscript was provided through an unrestricted educational grant from the California Dried Plum Board.

Author Contributions: T.C.W. analyzed data and wrote the manuscript.

Conflicts of Interest: The California Dried Plum Board had no role in the design of the study; in the collection, analyses or interpretation of data; in the writing of the manuscript; nor in the decision to publish the results. T.C.W. recused himself of all editorial involvement in the manuscript's review process.

\section{References}

1. US Department of Health and Human Services and US Department of Agriculture. 2015-2020 Dietary Guidelines for Americans. Available online: http://health.gov/dayietaryguidelines/2015/guidelines (accessed on 15 March 2017).

2. Wright, N.C.; Looker, A.C.; Saag, K.G.; Curtis, J.R.; Delzell, E.S.; Randall, S.; Dawson-Hughes, B. The recent prevalence of osteoporosis and low bone mass in the United States based on bone mineral density at the femoral neck or lumbar spine. J. Bone Min. Res. 2014, 29, 2520-2526. [CrossRef] [PubMed]

3. Looker, A.C.; Borrud, L.G.; Dawson-Hughes, B.; Shepherd, J.A.; Wright, N. Osteoporosis or low bone mass at the femur neck or lumbar spine in older adults: United States, 2005-2008. NCHS Data Brief 2012, 93, 1-8.

4. Burge, R.; Dawson-Hughes, B.; Solomon, D.H.; Wong, J.B.; King, A.; Tosteson, A. Incidence and economic burden of osteoporosis-related fractures in the United States, 2005-2025. J. Bone Min. Res. 2007, 22, 465-475. [CrossRef] [PubMed]

5. Topp, L.F.; Russell, D.M.; Neumuller, M.; Dalbo, M.A.; Liu, W. Plum. In Fruit Breeding; Springer: New York, NY, USA, 1991.

6. Food and Agriculture Organization of the United Nations. FAOSTAT 2013. Available online: http://faostat. fao.org (accessed on 27 October 2016).

7. US Department of Agriculture, Foreign Agriculture Service. Data \& Analysis. Available online: https: / / www.fas.usda.gov/data (accessed on 29 March 2016).

8. Tinker, L.F.; Schneeman, B.O.; Davis, P.A.; Gallaher, D.D.; Waggoner, C.R. Consumption of prunes as a source of dietary fiber in men with milk hypercholesterolemia. Am. J. Clin. Nutr. 1991, 53, 1259-1265. [PubMed]

9. Rothwell, J.A.; Perez-Jimenez, J.; Vos, F.; Crespy, V.; du Chaffaut, L.; Mennen, L.; Knox, C.; Eisner, R.; Cruz, J.; Wishart, D.; et al. Phenol-Explorer 3.0: A Major Update of the Phenol-Explorer Database to Incorporate Data on the Effects of Food Processing on Polyphenol Content. Database. Available online: http://phenol-explorer.eu/contents/food/51 (accessed on 15 March 2017).

10. Hooshmand, S.; Chai, S.C.; Saadat, R.L.; Payton, M.E.; Brummel-Smith, K.; Arjmandi, B.H. Comparative effects of dried plum and dried apple on bone in postmenopausal women. Br. J. Nutr. 2011, 106, 923-930. [CrossRef] [PubMed]

11. Hooshmand, S.; Kern, M.; Metti, D.; Shamloufard, P.; Chai, S.C.; Johnson, S.A.; Payton, M.E.; Arjmandi, B.H. The effect of two doses of dried plum on bone density and bone biomarkers in osteopenic postmenopausal women: A randomized, controlled trial. Osteoporos. Int. 2016, 27, 2271-2279. [CrossRef] [PubMed]

12. Arjmandi, B.H.; Khalil, D.A.; Lucas, E.A.; Georgis, A.; Stoecker, B.J.; Hardin, C.; Payton, M.E.; Wild, R.A. Dried plums improve indices of bone formation in postmenopausal women. J. Womens Health Gend Based Med. 2002, 11, 61-68. [CrossRef] [PubMed]

13. Hooshmand, S.; Brisco, J.R.Y.; Arjmandi, B.H. The effect of dried plum on serum levels of receptor activator of NF-kappaB ligand, osteoprotegerin and sclerostin in osteopenic postmenopausal women: A randomised controlled trial. Br. J. Nutr. 2014, 112, 55-60. [CrossRef] [PubMed]

14. Simonavice, E.; Liu, P.-Y.; Ilich, J.Z.; Kim, J.-S.; Arjmandi, B.; Panton, L.B. The effects of a 6-month resistance training and dried plum consumption intervention on strength, body composition, blood markers of bone turnover, and inflammation in breast cancer survivors. Appl. Physiol. Nutr. Metab. 2014, 39, 730-739. [CrossRef] [PubMed]

15. Arjmandi, B.H.; Johnson, C.D.; Campbell, S.C.; Hooshmand, S.; Chai, S.C.; Akhter, M.P. Combining fructooligosaccharide and dried plum has the greatest effect on restoring bone mineral density among select functional foods and bioactive compounds. J. Med. Food 2010, 13, 312-319. [CrossRef] [PubMed] 
16. Bu, S.Y.; Lucas, E.A.; Franklin, M.; Marlow, D.; Brackett, D.J.; Boldrin, E.A.; Devareddy, L.; Arjmandi, B.H.; Smith, B.J. Comparison of dried plum supplementation and intermittent PTH in restoring bone in osteopenic orchidectomized rats. Osteoporos. Int. 2007, 18, 931-942. [CrossRef] [PubMed]

17. Deyhim, F.; Stoecker, B.J.; Brusewitz, G.H.; Devareddy, L.; Arjmandi, B.H. Dried plum reverses bone loss in an osteopenic rat model of osteoporosis. Menopause 2005, 12, 755-762. [CrossRef] [PubMed]

18. Franklin, M.; Bu, S.Y.; Lerner, M.R.; Lancaster, E.A.; Bellmer, D.; Marlow, D.; Lightfoot, S.A.; Arjmandi, B.H.; Brackett, D.J.; Lucas, E.A. Dried plum prevents bone loss in a male osteoporosis model via IGF-I and the RANK pathway. Bone 2006, 39, 1331-1342. [CrossRef] [PubMed]

19. Halloran, B.P.; Wronski, T.J.; VonHerzen, D.C.; Chu, V.; Xia, X.; Pingel, J.E.; Williams, A.A.; Smith, B.J. Dietary dried plum increases bone mass in adult and aged male mice. J. Nutr. 2010, 140, 1781-1787. [CrossRef] [PubMed]

20. Johnson, C.D.; Lucas, E.A.; Hooshmand, S.; Campbell, S.; Akhter, M.P.; Arjmandi, B.H. Addition of fructooligosaccharides and dried plum to soy-based diets reverses bone loss in the ovariectomized rat. Evid. Based Complement. Alternat. Med. 2011. [CrossRef] [PubMed]

21. Leotoing, L.; Wauquier, F.; Davicco, M.-J.; Lebecque, P.; Gaudout, D.; Rey, S.; Vitrac, X.; Massenat, L.; Rashidi, S.; Wittrant, Y. The phenolic acids of Agen prunes (dried plums) or Agen prune juice concentrates do not account for the protective action on bone in a rat model of postmenopausal osteoporosis. Nutr. Res. 2016, 36, 161-173. [CrossRef] [PubMed]

22. Monsefi, M.; Parvin, F.; Farzaneh, M. Effects of plum extract on skeletal system of fetal and newborn mice. Med. Princ. Pract. 2013, 22, 351-356. [CrossRef] [PubMed]

23. Pawlowski, J.W.; Martin, B.R.; McCabe, G.P.; Ferruzzi, M.G.; Weaver, C.M. Plum and soy aglycon extracts superior at increasing bone calcium retention in ovariectomized Sprague Dawley rats. J. Agric. Food Chem. 2014, 62, 6108-6117. [CrossRef] [PubMed]

24. Rendina, E.; Lim, Y.F.; Marlow, D.; Wang, Y.; Clarke, S.L.; Kuvibidila, S.; Lucas, E.A.; Smith, B.J. Dietary supplementation with dried plum prevents ovariectomy-induced bone loss while modulating the immune response in C57BL/6J mice. J. Nutr. Biochem. 2012, 23, 60-68. [CrossRef] [PubMed]

25. Rendina, E.; Hembree, K.D.; Davis, M.R.; Marlow, D.; Clarke, S.L.; Halloran, B.P.; Lucas, E.A.; Smith, B.J. Dried plum's unique capacity to reverse bone loss and alter bone metabolism in postmenopausal osteoporosis model. PLoS ONE 2013, 8, e60569. [CrossRef] [PubMed]

26. Schreurs, A.-S.; Shirazi-Fard, Y.; Shahnazari, M.; Alwood, J.S.; Truong, T.A.; Tahimic, C.G.T.; Limoli, C.L.; Turner, N.D.; Halloran, B.; Globus, R.K. Dried plum diet protects from bone loss caused by ionizing radiation. Sci. Rep. 2016, 6, 21343. [CrossRef] [PubMed]

27. Shahnazari, M.; Turner, R.T.; Iwaniec, U.T.; Wronski, T.J.; Li, M.; Ferruzzi, M.G.; Nissenson, R.A.; Halloran, B.P. Dietary dried plum increases bone mass, suppresses proinflammatory cytokines and promotes attainment of peak bone mass in male mice. J. Nutr. Biochem. 2016, 34, 73-82. [CrossRef] [PubMed]

28. Smith, B.J.; Bu, S.Y.; Wang, Y.; Rendina, E.; Lim, Y.F.; Marlow, D.; Clarke, S.L.; Cullen, D.M.; Lucas, E.A. A comparative study of the bone metabolic response to dried plum supplementation and PTH treatment in adult, osteopenic ovariectomized rat. Bone 2014, 58, 151-159. [CrossRef] [PubMed]

29. Smith, B.J.; Graef, J.L.; Wronski, T.J.; Rendina, E.; Williams, A.A.; Clark, K.A.; Clarke, S.L.; Lucas, E.A.; Halloran, B.P. Effects of dried plum supplementation on bone metabolism in adult C57BL/6 male mice. Calcif. Tissue Int. 2014, 94, 442-453. [CrossRef] [PubMed]

30. Arjmandi, B.H.; Lucas, E.A.; Juma, S.; Soliman, A.; Stoecker, B.J.; Khalil, D.A.; Smith, B.J.; Wang, C. Dried plums prevent ovariectomy-induced bone loss in rats. J. Am. Nutraceut. Assoc. 2001, 4, 50-56.

31. Bu, S.Y.; Lerner, M.; Stoecker, B.J.; Boldrin, E.; Brackett, D.J.; Lucas, E.A.; Smith, B.J. Dried plum polyphenols inhibit osteoclastogenesis by downregulating NFATc1 and inflammatory mediators. Calcif. Tissue Int. 2008, 8, 475-488. [CrossRef] [PubMed]

32. Bu, S.Y.; Hunt, T.S.; Smith, B.J. Dried plum polyphenols attenuate the detrimental effects of TNF-alpha on osteoblast function coincident with up-regulation of Runx2, Osterix and IGF-I. J. Nutr. Biochem. 2009, 20, 35-44. [CrossRef] [PubMed]

33. Hooshmand, H.; Kumar, A.; Zhang, J.Y.; Johnson, S.A.; Chai, S.C.; Arjmandi, B.H. Evidence for anti-inflammatory and antioxidative properties of dried plum polyphenols in macrophage RAW 264.7 cells. Food Funct. 2015, 6, 1719-1725. [CrossRef] [PubMed] 
34. Barone, C.M.; Marion, R.; Shanske, A.; Argamaso, R.V.; Shprintzen, R.J. Craniofacial, limb, and abdominal anomalies in a distinct syndrome: Relation to the spectrum of Pfeiffer syndrome type 3. Am. J. Med. Genet. 1993, 45, 745-750. [CrossRef] [PubMed]

35. Bracero, L.A.; Clark, D.; Pieffer, M.; Fakhry, J. Sonographic findings in a case of cloverleaf skull deformity and prune belly. Am. J. Perinatol. 1988, 5, 239-241. [CrossRef] [PubMed]

36. Frydman, M.; Cohen, H.A.; Ashkenazi, A.; Varsano, I. Familial segregation of cervical ribs, Sprengel anomaly, preaxial polydactyly, anal atresia, and urethral obstruction: A new syndrome? Am. J. Med. Genet. 1993, 45, 717-720. [CrossRef] [PubMed]

37. Fuentes, J.M.; Bouscarel, C.; Choucair, Y.; Roquefeuil, B.; Vlahovitch, B.; Blanchet, P. Monitoring of intracranial pression in acute neurotrauma by extra-dural screw (author's transl). Anesth. Analg. 1979, 36, 429-433.

38. Gambacorta, D.; Biancotti, R.; Consorti, P.; Zei, E. Clinical aspects of the prognosis of skull and brain injuries. A study of 108 cases. Minerva Anestesiol. 1980, 46, 703-708. [PubMed]

39. Gearhart, J.P.; Albertsen, P.C.; Marshall, F.F.; Jeffs, R.D. Pediatric applications of augmentation cystoplasty: The Johns Hopkins experience. J. Urol. 1986, 136, 430-432. [PubMed]

40. Gofton, W.T.; Macdermid, J.C.; Patterson, S.D.; Faber, K.J.; King, G.J.W. Functional outcome of AO type C distal humeral fractures. J. Hand Surg. Am. 2003, 28, 294-308. [CrossRef] [PubMed]

41. Hooshmand, S.; Arjmandi, B.H. Viewpoint: Dried plum, an emerging functional food that may effectively improve bone health. Ageing Res. Rev. 2009, 8, 122-127. [CrossRef] [PubMed]

42. Hublin, J.J. Paleoanthropology: Homo erectus and the limits of a paleontological species. Curr. Biol. 2014, 24, R82-R84. [CrossRef] [PubMed]

43. Kostka, V.M.; Krautwald-Junghanns, M.E.; Balks, E. Polyostotic hyperostosis in a plum-headed parakeet (Psittacula cyanocephala L., 1766). Tierarztl. Prax 1996, 24, 36-40. [PubMed]

44. Loh, K.C.; Salisbury, S.R.; Accott, P.; Gillis, R.; Crocker, J.F. Central precocious puberty and chronic renal failure: A reversible condition post renal transplantation. J. Pediatr. Endocrinol. Metab. 1997, 10, 539-545. [CrossRef] [PubMed]

45. Oostenbroek, H.J.; Brand, R.; van Roermund, P.M. Lower limb deformity due to failed trauma treatment corrected with the Ilizarov technique: Factors affecting the complication rate in 52 patients. Acta Orthop. 2009, 80, 435-439. [CrossRef] [PubMed]

46. Pagon, R.A.; Smith, D.W.; Shepard, T.H. Urethral obstruction malformation complex: A cause of abdominal muscle deficiency and the "prune belly". J. Pediatr. 1979, 94, 900-906. [CrossRef]

47. Robicsek, F.; Watts, L.T. Pectus carinatum. Thorac. Surg. Clin. 2010, 20, 563-574. [CrossRef] [PubMed]

48. Romero Otero, J.; Gomez Fraile, A.; Feltes Ochoa, J.; Blanco Carballo, O.; Aransay Bramtot, A.; Lopez Vazquez, F.; Alonso, L. Megalourethra in association with VACTER syndrome. Actas Urol. Esp. 2006, 30, 412-414. [CrossRef]

49. Sacco, S.M.; Horcajada, M.-N.; Offord, E. Phytonutrients for bone health during ageing. Br. J. Clin. Pharmacol. 2013, 75, 697-707. [CrossRef] [PubMed]

50. Shah, D.; Sharma, S.; Faridi, M.M.A.; Mishra, K. VACTERL association with Prune-Belly syndrome. Indian Pediatr. 2004, 41, 845-847. [PubMed]

51. Shamberger, R.C.; Welch, K.J. Surgical repair of pectus excavatum. J. Pediatr. Surg. 1988, $23,615-622$. [CrossRef]

52. Shen, C.-L.; von Bergen, V.; Chyu, M.-C.; Jenkins, M.R.; Mo, H.; Chen, C.-H.; Kwun, I.-S. Fruits and dietary phytochemicals in bone protection. Nutr. Res. 2012, 32, 897-910. [CrossRef] [PubMed]

53. Shen, W.; Wang, G.; Cui, J.; He, J. Skull plasty to correct congenital craniosynostosis. Zhonghua Zheng Xing Wai Ke Za Zhi 2007, 23, 284-287. [PubMed]

54. Shen, W.; Wang, G.; Cui, J.; He, J.; Chen, J. Cranial vault reconstruction of plagiocephaly. Zhonghua Zheng Xing Wai Ke Za Zhi 2007, 23, 459-462. [PubMed]

55. Shen, W.; Wang, G.; Wu, Y.; Cui, J.; He, J. Total calvarial reconstruction for sagittal synostosis. Zhonghua Zheng Xing Wai Ke Za Zhi 2006, 22, 172-174. [PubMed]

56. Smolkin, T.; Soudack, M.; Goldstein, I.; Sujov, P.; Makhoul, I.R. Prune belly syndrome: Expanding the phenotype. Clin. Dysmorphol. 2008, 17, 133-135. [CrossRef] [PubMed]

57. Stacewicz-Sapuntzakis, M. Dried plums and their products: Composition and health effects-An updated review. Crit. Rev. Food Sci. Nutr. 2013, 53, 1277-1302. [CrossRef] [PubMed] 
58. Wang, L.; Gao, W.; Xiong, K.; Hu, K.; Liu, X.; He, H. VEGF and BFGF expression and histological characteristics of the bone-tendon junction during acute injury healing. J. Sports Sci. Med. 2014, 13, 15-21. [PubMed]

59. Wheeler, P.G.; Weaver, D.D. Adults with VATER association: Long-term prognosis. Am. J. Med. Genet. Part A 2005, 138A, 212-217. [CrossRef] [PubMed]

60. Zeng, H.; Liu, Y. Electro-acupuncture combined with plum-blossom needle tapping for treatment of supraorbital neuritis-a clinical observation of 59 cases. J. Tradit. Chin. Med. 2003, 23, 193-194. [PubMed]

61. Zhao, R.; Liu, Z.; Wang, J.; Xie, G. Combination of acupuncture with cupping increases life quality of patients of osteoporosis. Zhongguo Zhen Jiu 2008, 28, 873-875. [PubMed]

62. Weaver, C.M.; Alekel, D.L.; Ward, W.E.; Ronis, M.J. Flavonoid intake and bone health. J. Nutr. Gerontol. Geriatr. 2012, 31, 239-253. [CrossRef] [PubMed]

63. Vasikaran, S.; Eastell, R.; Bruyere, O.; Foldes, A.J.; Garnero, P.; Griesmacher, A.; McClung, M.; Morris, H.A.; Silverman, S.S.; Trenti, T.; et al. Markers of bone turnover for the prediction of fracture risk and monitoring of osteoporosis treatment: a need for international reference standards. Osteoporos. Int. 2011, 22, 391-420. [CrossRef] [PubMed]

(C) 2017 by the author. Licensee MDPI, Basel, Switzerland. This article is an open access article distributed under the terms and conditions of the Creative Commons Attribution (CC BY) license (http:/ / creativecommons.org/licenses/by/4.0/). 